\title{
Deformed Topological Partition Function and Nekrasov Backgrounds
}

\author{
I. Antoniadis*, S. Hohenegger ${ }^{\dagger}$, K.S. Narain ${ }^{\ddagger}$, T.R. Taylor ${ }^{\S}$ \\ *Department of Physics, CERN - Theory Division, CH-1211 Geneva 23, Switzerland \\ ${ }^{\dagger}$ Institut für Theoretische Physik, ETH Zürich, CH-8093 Zürich, Switzerland \\ ${ }^{\ddagger}$ High Energy Section, The Abdus Salam International Center for Theoretical Physics, \\ Strada Costiera, 11-34014 Trieste, Italy \\ $\S$ Center for Advanced Studies, Ludwig-Maximilians-Universität, \\ Seestraße 13, 80802 Munich, Germany
}

\begin{abstract}
A deformation of the $\mathcal{N}=2$ topological string partition function is analyzed by considering higher dimensional F-terms of the type $W^{2 g} \Upsilon^{n}$, where $W$ is the chiral Weyl superfield and each $\Upsilon$ factor stands for the chiral projection of a real function of $\mathcal{N}=2$ vector multiplets. These terms generate physical amplitudes involving two anti-self-dual Riemann tensors, $2 g-2$ anti-self-dual graviphoton field strengths and $2 n$ self-dual field strengths from the matter vector multiplets. Their coefficients $F_{g, n}$ generalizing the genus $g$ partition function $F_{g, 0}$ of the topological twisted type II theory, can be used to define a generating functional by introducing deformation parameters besides the string coupling. Choosing all matter field strengths to be that of the dual heterotic dilaton supermultiplet, one obtains two parameters that we argue should correspond to the deformation parameters of the Nekrasov partition function in the field theory limit, around the conifold singularity. Its perturbative part can be obtained from the one loop analysis on the heterotic side. This has been computed in [1] and in the field theory limit shown to be given by the radius deformation of $c=1 \mathrm{CFT}$ coupled to two-dimensional gravity. Quite remarkably this result reproduces the gauge theory answer up to a phase difference that may be attributed to the regularization procedure. The type II results are expected to be exact and should also capture the part that is non-perturbative in heterotic dilaton.
\end{abstract}

\footnotetext{
*ignatios.antoniadis@cern.ch. On leave from CPHT (UMR CNRS 7644) Ecole Polytechnique, F-91128 Palaiseau.

${ }^{\dagger}$ stefanh@itp.phys.ethz.ch

${ }^{\dagger}$ narain@ictp.trieste.it

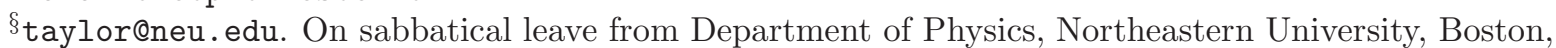
MA 02115, U.S.A.
} 


\section{Introduction}

$\mathcal{N}=2$ supersymmetric theories provide an interesting arena for studying non perturbative dynamics of field and string theories (in the absence or presence of gravity), exhibiting a remarkable web of dualities among very different systems. It was initiated with the computation of the prepotential that describes the exact two derivative effective action in the Coulomb phase of $\mathcal{N}=2$ super Yang Mills theory [2]. This result was subsequently generalized in the presence of gravity [3], using string duality between heterotic compactifications on $K 3 \times T^{2}$, that describe the perturbative expansion around an enhanced symmetric point of the gauge symmetry, and type II on a Calabi-Yau threefold which is an appropriate $K 3$ fibration, that provides the exact answer around the conifold singularity where massless charged hypermultiplets appear as non perturbative states [4]. The field theory limit corresponds to the double scaling where the string length and the distance from the conifold point go to zero, keeping fixed the dynamical Yang Mills scale.

On the other hand, it was known that the prepotential is the first of a series of $\mathcal{N}=2 \mathrm{~F}$ terms of the type $F_{g} W^{2 g}$ where $W$ is the chiral Weyl superfield, containing the graviphoton anti-self-dual field strength as the lowest component, and $F_{g}$ are analytic functions of the vector moduli [5, 6]; they are computed by the genus $g$ partition function of the twisted Calabi-Yau sigma model and have a non analytic part due to a holomorphic anomaly that satisfies an equation which provides interesting recursion relations. Moreover, their behavior near the conifold singularity is governed by $c=1$ string theory at the self-dual radius [7, 8]. This was explicitly checked on the heterotic side, where $F_{g}$ 's start receiving contributions already at one loop [9]. It was indeed shown that near the enhanced symmetry point the leading singularity of $F_{g}$ (for $g>1$ ) is a universal pole of order $2 g-2$ with a coefficient given by the Euler number of the genus- $g$ Riemann surface.

Another important result was the explicit computation of the $\mathcal{N}=2$ gauge theory partition function [10] by performing the sum over all instanton contributions. This was achieved by calculating the instanton partition function as a function of two complex parameters $\epsilon_{1,2}$ that regulate the integral over the instanton moduli space, and interpreting it as a six-dimensional theory in the so-called $\Omega$ supergravity background [11, 12, It was then shown that upon extracting a divergent volume factor $1 / \epsilon_{1} \epsilon_{2}$, one can reproduce the Seiberg-Witten prepotential in the limit $\epsilon_{1,2} \rightarrow 0$. Moreover, it was argued that all higher $F_{g}$ 's can be reproduced in the field theory limit, by identifying $\epsilon_{1}=-\epsilon_{2}$ with the topological string coupling.

The perturbative part of the gauge theory for $\epsilon_{+} \equiv\left(\epsilon_{1}+\epsilon_{2}\right) / 2=0$ was exactly the same as the leading terms near the $S U(2)$ enhancement in the one loop heterotic result for the generating function $\sum_{g} \epsilon_{-}^{2 g} F_{g}[9]$. In fact the latter was just given by the Schwinger like formula for a one loop diagram for states that become massive in the Coulomb branch of $\mathcal{N}=2 S U(2)$ gauge theory, coupled to a constant anti-self dual background field strength. In the Gopakumar-Vafa reformulation [13, 14] of the topological partition function the generating function of the A-model $F_{g}$ is reproduced by integrating all the massive BPS states which are the D-brane states wrapping on 2-cycles of the Calabi-Yau space in the background of a constant anti-self dual graviphoton field strength; the contribution of 
each of these states being given by the Schwinger formula. Due to anti-self duality, the graviphoton field strength couples to the spin of the D-brane states in one of the $S U(2)$ in the $S O(4)$ Lorentz group. Using the technique of the topological vertex for toric noncompact Calabi-Yau spaces that can be used to geometrically engineer gauge theories, it was shown in [15] that $F_{g}$ captures also the non-perturbative part of the gauge theory for $\epsilon_{1}=-\epsilon_{2}$ where the combination $\epsilon_{1}-\epsilon_{2}$ has the interpretation of the background of the anti-self dual graviphoton field strength. This suggests that $\epsilon$ 's are not just regularization parameters and opens the question what is the string theory interpretation of the second parameter $\epsilon_{+}$.

In [16], a generalization of the Gopakumar-Vafa formula was proposed which includes coupling to spins of both $S U(2)$ of the D-brane states. The couplings correspond to backgrounds involving both self-dual and anti-self dual gauge field strengths. Using the formulation of a refined topological vertex for non compact toric Calabi-Yau spaces it was shown that this generalized formula reproduces the non-perturbative part of the gauge theory result for arbitrary $\epsilon_{-}$and $\epsilon_{+}$. This raises the question what string theory amplitude gives rise to this generalized Gopakumar-Vafa formula and which string effective action term it computes.

In this work, we address the above question by studying possible deformations of the topological string and we make a proposal based on some plausible arguments. Indeed, the $\Omega$-background for the six-dimensional metric corresponds to constant field strengths for some Kaluza-Klein gauge fields that include the $\mathcal{N}=2$ graviphoton, as well as another direction, such that part of the supersymmetry is preserved. This leads us to consider $\mathcal{N}=2$ higher dimensional F-terms that generalize the series $F_{g} W^{2 g}$ and involve only vector multiplets. By analogy with a similar study which has been performed in the past for $\mathcal{N}=1$ amplitudes [17, we consider a 'dressing' of $W^{2 g}$ with chiral projections of functions of vector multiplets that we call generically $\Upsilon: W^{2 g} \Upsilon^{n}$. These terms generate, in particular, amplitudes of the form $F_{g, n}\left(R^{-}\right)^{2}\left(T^{-}\right)^{2 g-2}\left(F^{+}\right)^{2 n}$, where $R$ denotes the Riemann tensor, $T$ the graviphoton, $F$ the remaining matter gauge field strengths, while the superscripts - and + denote their anti-self-dual and self-dual projections, respectively. We have here suppressed the anti-holomorphic vector moduli indices both in the matter field strengths $F$ as well as in the coefficient $F_{g, n}$ which depend on both holomorphic and anti-holomorphic vector moduli 1 On the type II side, these amplitudes are topological, given essentially by the square of the left-moving (supersymmetric) part of the corresponding heterotic higher dimensional terms [17].

Motivated again by the $\Omega$-background, we are led to consider the case where all matter gauge field strengths are those of the heterotic dilaton vector supermultiplet, arising from compactification of the $\mathcal{N}=1$ six-dimensional theory on $T^{2}$. Such amplitudes have been considered before in [1] and were studied on the heterotic side. Their behavior near the enhanced symmetric point was found to be determined by the radius deformation of the

\footnotetext{
${ }^{1}$ The dependence of $F_{g, n}$ on the anti-holomorphic vector moduli is controlled by certain differential equations that arise from their origin in the F-term [17. We leave a discussion of these classical equations as well as the possible anomalies at the string level for future.
} 
$c=1$ string theory. Here, we compare their expression with the perturbative limit of the Nekrasov partition function and we find a remarkable agreement up to a phase factor that could be attributed to a difference in the field theory regularization. More precisely, the string theory amplitudes in the heterotic perturbative limit are invariant under parity which changes the sign of one of the two $\epsilon_{1}$ or $\epsilon_{2}$ but leaves the other invariant. On the other hand, the field theory result breaks the symmetry due to the additional phase, although the corresponding action is invariant [18], up to the $\theta$-term which is relevant only for non-perturbative contributions.

The plan of this paper is the following. In Section 2, we define the new amplitudes and describe the effective Lagrangian. Then, we compute the corresponding amplitudes in string theory on the type II side (Section 3) and review the corresponding heterotic computation of [1] in Section 4. In type II theory, they are topological and are expected to be exact at genus $g$, while on the heterotic side they start receiving contributions at one loop. Finally, we take the field theory limit near the enhanced symmetric point and compare the resulting expression with the perturbative part of Nekrasov's partition function.

\section{Higher Derivative Couplings in the String Effective Action}

In this section we discuss a class of amplitudes in $\mathcal{N}=2$ compactifications of string theory, which we will compare to the Nekrasov-partition function as discussed in [10, 11, 12, 18]. In [5] a particular class of higher derivative chiral F-terms

$$
\mathcal{I}_{g}=\int d^{4} x \int d^{4} \theta F_{g}(X) W^{2 g}
$$

in the effective $\mathcal{N}=2$ Poincaré supergravity action has been considered. Here $W$ is the Weyl superfield which has Weyl weight $w=1$. It has the following expansion (we only display the bosonic components)

$$
W_{\mu \nu}^{i j}=\epsilon^{i j} T_{\mu \nu}^{-}-R_{\mu \nu \lambda \rho}^{-}\left(\theta^{i} \sigma^{\lambda \rho} \theta^{j}\right)+\ldots
$$

where $T_{\mu \nu}^{-}$is the anti-self-dual graviphoton field strength and $R_{\mu \nu \lambda \rho}^{-}$is the anti-self-dual Riemann tensor. In this respect $\mu, \nu$ denote four-dimensional Lorentz indices, while $i, j \in$ $S U(2)_{R}$ are indices of the $\mathcal{N}=2$ R-symmetry group.

Furthermore, $F_{g}$ is an analytic homogeneous function of degree $(2-2 g)$ of the "reduced" $\mathcal{N}=2$ chiral superfields $X^{I}$

$$
X^{I}=\phi^{I}+\frac{1}{2} F_{\lambda \rho}^{-I} \epsilon_{i j}\left(\theta^{i} \sigma^{\lambda \rho} \theta^{j}\right)+\ldots
$$

which have Weyl weight $w=1$ and chiral weight $c=-1$. The field $X^{0}$ acts as a compensator and the unconstrained physical scalars (the moduli) are parameterized by $\hat{\phi}^{I}=\phi^{I} / \phi^{0}$ 
for $I=1, \ldots, h . F_{\lambda \rho}^{-I}$ are the corresponding anti-self-dual gauge field strengths. Notice in particular that the superfields

$$
\hat{X}^{I}=\frac{X^{I}}{X^{0}}
$$

(as well as arbitrary functions thereof) have vanishing Weyl and conformal weight. The functions $F_{g}$ have been shown to be computed by the partition function of the $\mathcal{N}=2$ topological string. Furthermore, in the field theory limit i.e. decoupling the gravitational sector apart from their backgrounds, the generating function $F(\lambda)=\sum_{g} \lambda^{2 g} F_{g}$ is related to the $\mathcal{N}=2$ gauge theory partition function in the presence of anti-self-dual graviphoton field strength $\left(\epsilon_{1}=-\epsilon_{2}\right.$ in the notation of [10, 18] $)$.

In this note we will consider a slight generalization of (2.1). To this end, we introduce the superconformal chiral projection operator (see e.g. [19])

$$
\Pi=\left(\epsilon_{i j} \bar{D}^{i} \bar{\sigma}_{\mu \nu} \bar{D}^{j}\right)^{2}, \quad \text { with } \quad \Pi\left(X^{I}\right)=0 \text { and } \Pi\left(X^{I}\right)^{\dagger}=96 \square X^{I} .
$$

From this relation we can read off that $\Pi$ has Weyl weight $w=2$ and conformal weight $c=-2$. Therefore, for an arbitrary function $G\left(\hat{X}^{I},\left(\hat{X}^{I}\right)^{\dagger}\right)$ the superfield

$$
\Upsilon=\Pi \frac{G\left(\hat{X}^{I},\left(\hat{X}^{I}\right)^{\dagger}\right)}{\left(X^{0}\right)^{2}}
$$

will have vanishing Weyl and conformal weights. Notice in particular that the first term in the expansion of $\Upsilon$ will be given by

$$
\Upsilon=\frac{\partial^{2} G\left(\hat{\phi}, \hat{\phi}^{\dagger}\right)}{\partial\left(\hat{\phi}^{I}\right)^{\dagger} \partial\left(\hat{\phi}^{J}\right)^{\dagger}}\left(F^{+I} F^{+J}\right)+\ldots
$$

We can therefore generalize (2.1) in the following way

$$
\mathcal{I}_{g, n}=\int d^{4} x \int d^{4} \theta W^{2 g} \Upsilon^{n} \sim \int d^{4} x F_{g, n}(\hat{\phi}, \hat{\bar{\phi}})\left(R^{-}\right)^{2}\left(T^{-}\right)^{2 g-2}\left(F^{+}\right)^{2 n}+\ldots
$$

Here we have suppressed the $2 n$ anti-holomorphic vector indices in $F_{g, n}$ that contract the corresponding indices on the $2 n$ matter gauge fields and the dots denote further terms, which will play no role in this note. The anti-holomorphic moduli dependence in $F_{g, n}$ comes from $\Upsilon$ as shown in (2.7).

We would like to point out that terms involving both anti-self-dual and self-dual graviphoton field strengths cannot be written as a chiral F-term. This is because the self-dual graviphoton field strength is the lowest component of the anti-chiral Weyl multiplet. Thus $n$ chiral projectors acting on functions of anti-chiral Weyl superfields will pick out $2 n$ self dual Riemann tensors. 


\section{The Amplitude in Type II String Theory}

We will now try to recover the higher derivative terms (2.8) in string theory, starting with type II compactified on an arbitrary Calabi-Yau manifold. The couplings (2.1) have been recovered as $g$-loop amplitudes in [5]. Since (by construction) the superfields $\Upsilon$ which we have added in (2.8) have no Weyl weight, we are led to conclude that also $F_{g, n}$ corresponds to a $g$-loop amplitude in the type II theory. We will now try to explicitly verify this conjecture.

General expressions for the vertex operators which are needed to compute the amplitude corresponding to the component term that we have explicitly displayed in (2.8) can for example be found in [5]. Taking into account that the gauge fields are self-dual in (2.8), while the Riemann tensors and graviphotons are anti-self-dual, we are led to specifically consider the following amplitude

$$
F_{g, n}=\left\langle V_{(R)}\left(p_{1}\right) V_{(R)}\left(\bar{p}_{1}\right) \prod_{i=1}^{g-1} V_{(T)}^{(-1 / 2)}\left(\bar{p}_{1}^{i}\right) V_{(T)}^{(-1 / 2)}\left(\bar{p}_{1}^{i}\right) \prod_{j=1}^{n} V_{(F), I_{j}}^{(-1 / 2)}\left(p_{1}^{j}\right) V_{(F), J_{j}}^{(-1 / 2)}\left(\bar{p}_{1}^{j}\right)\right\rangle_{\text {g-loop }}^{\text {type II }},
$$

where we have picked the following helicity and momentum configurations in the various vertices

$$
\begin{array}{ll}
\text { gravitons: } & V_{(R)}\left(p_{1}\right)=:\left(\partial Z^{2}-i p_{1} \chi^{1} \chi^{2}\right)\left(\bar{\partial} Z^{2}-i p_{1} \tilde{\chi}^{1} \tilde{\chi}^{2}\right) e^{i p_{1} Z^{1}}\left(z_{1}, \bar{z}_{1}\right):, \\
& V_{(R)}\left(\bar{p}_{1}\right)=:\left(\partial \bar{Z}^{2}-i \bar{p}_{1} \bar{\chi}^{1} \bar{\chi}^{2}\right)\left(\bar{\partial} \bar{Z}^{2}-i \bar{p}_{1} \overline{\tilde{\chi}}^{1} \tilde{\tilde{\chi}}^{2}\right) e^{i \bar{p}_{2} \bar{Z}^{2}}\left(z_{2}, \bar{z}_{2}\right):, \\
\text { graviphotons: } & V_{(T)}^{(-1 / 2)}\left(p_{1}\right)=: p_{1} e^{-\frac{1}{2}(\varphi+\tilde{\varphi})} S_{1} \tilde{S}_{1} \Sigma(x, \bar{x}) e^{i p_{1} Z^{1}}:, \\
& V_{(T)}^{(-1 / 2)}\left(\bar{p}_{1}\right)=: \bar{p}_{1} e^{-\frac{1}{2}(\varphi+\tilde{\varphi})} S_{2} \tilde{S}_{2} \Sigma(y, \bar{y}) e^{i \bar{p}_{1} \bar{Z}^{1}}:, \\
\text { gauge fields: } \quad & V_{(F), I}^{(-1 / 2)}\left(p_{1}\right)=: p_{1} e^{-\frac{1}{2}(\varphi+\tilde{\varphi})} S^{1} \tilde{S}^{1} \Sigma_{I}(u, \bar{u}) e^{i p_{1} Z^{1}}: \\
& V_{(F), J}^{(-1 / 2)}\left(\bar{p}_{1}\right)=: c \tilde{c} \bar{p}_{1} e^{-\frac{1}{2}(\varphi+\tilde{\varphi})} S^{\dot{2}} \tilde{S}^{\dot{2}} \Sigma_{J}(v, \bar{v}) e^{i \bar{p}_{1} \bar{Z}^{1}}: .
\end{array}
$$

Here we have adopted the convention to denote right moving degrees of freedom with a tilde. We have introduced a complex basis for the space-time (Euclidean) coordinates $\left(Z^{1}, \bar{Z}^{1}, Z^{2}, \bar{Z}^{2}\right)$ as well as their fermionic partners $\left(\chi^{1}, \bar{\chi}^{1}, \chi^{2}, \bar{\chi}^{2}\right)$. If we bosonize the latter in terms of $\phi_{1,2}$ we can write for the space-time spin fields

$$
\begin{aligned}
& S_{1}=e^{\frac{i}{2}\left(\phi_{1}+\phi_{2}\right)}, \quad \text { and } \quad S_{2}=e^{-\frac{i}{2}\left(\phi_{1}+\phi_{2}\right)} \text {, } \\
& S^{\dot{1}}=e^{\frac{i}{2}\left(\phi_{1}-\phi_{2}\right)}, \quad \text { and } \quad S^{\dot{2}}=e^{-\frac{i}{2}\left(\phi_{1}-\phi_{2}\right)} \text {. }
\end{aligned}
$$

Recall that the self-dual and anti-self-dual tensors transform under the Euclideanized Lorentz group $S O(4)=S U(2) \times S U(2)$ as $(3,1)$ and $(1,3)$ representations respectively. For the gauge field vertices above we have chosen the helicity combination $S^{i} \tilde{S}^{i}$ and $S^{\dot{2}} \tilde{S}^{\dot{2}}$ which corresponds to helicity +1 and -1 of the corresponding $S U(2)$. We have distinguished the 
vector indices for these two helicities by the indices $I_{i}$ and $J_{j}$ with $i, j=1, \ldots, n$ respectively. Generalization to the helicity 0 would be given by the vertices $S^{(i} \tilde{S}^{\dot{2})}$ and an analysis for this case can also be carried out. At the end we will point out the resulting modification. Note that when one of the vertices labelled by index $I$ approaches another labelled by $J$, they could form a Lorentz invariant combination and could result in a divergence associated with the reducible graph connecting the gauge kinetic term $F_{I} \cdot F_{J}$ with the amplitude $F_{g, n-1}$ via the exchange of a scalar modulus (see Figure 1). We will comment on this further at the end of this section.

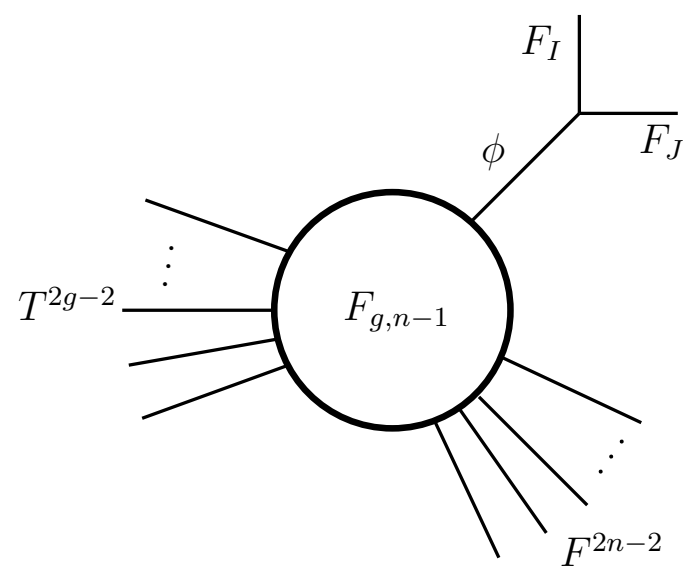

Figure 1: Schematic drawing of the reducible diagram: The gauge kinetic term $F_{I} \cdot F_{J}$ is connected to $F_{g, n-1}$ via the exchange of the scalar $\phi$.

$\Sigma$ is a field in the internal $\mathcal{N}=2$ sector with $U(1)$-charges $(3 / 2,-3 / 2)$ in the type IIA theory and $(3 / 2,3 / 2)$ in type IIB. Bosonizing the $U(1)$-current $J$ of the internal $\mathcal{N}=2$ $\mathrm{SCFT}^{2}$ by $H$ we can explicitly write

$$
\Sigma(z, \bar{z})=e^{\frac{i \sqrt{3}}{2}(H(z) \mp \tilde{H}(\bar{z}))},
$$

where the upper sign corresponds to IIA and the lower to IIB (for further details see e.g. [5]). The operators $\Sigma_{I}$ have charge $(1 / 2,-1 / 2)[(1 / 2,1 / 2)]$ in type IIA (type IIB) respectively and are given by

$$
\Sigma_{I}(z, \bar{z})=\lim _{w \rightarrow z}|w-z| e^{\frac{\sqrt{3} i}{2}(H(w) \mp \tilde{H}(\bar{w}))} \Psi_{I}(z, \bar{z}),
$$

where $\Psi_{I}$ are (anti chiral, chiral) (or (anti chiral, anti-chiral)) primary operators in IIA (IIB). Besides this, observe that we have put $c$-ghosts together with half of the vertices of the $\mathcal{N}=2$ matter vectors. Because of this, the corresponding positions will not be

\footnotetext{
${ }^{2}$ The full SCFT will be spanned by the energy momentum tensor $T$, two spin-3/2 supercurrents $G^{ \pm}$ and the $U(1)$-current $J$.
} 
integrated over the world-sheet, but instead we will have $n$ additional Beltrami-differentials $\mu$ corresponding to the punctures.

Finally, the graviton vertices are written in the (0)-ghost picture while all remaining vertices are in the $(-1 / 2)$-picture which necessitates the insertion of $(g-1+n)$ picture changing operators $(\mathrm{PCO})$. Taking into account that we need to insert further $(2 g-2)$ $\mathrm{PCO}$ which arise after integrating out the superghosts, the total number of PCO will be $3 g-3+n$ and they will be inserted at world-sheet positions $r_{a=1, \ldots, 3 g-3+n}$.

After these preliminaries we will now extract the piece of the correlator which is proportional to $p_{1}^{2} \bar{p}_{1}^{2} \prod_{i=1}^{g-1} \bar{p}_{1}^{i} \bar{p}_{1}^{i} \prod_{j=1}^{n} p_{1}^{j} \bar{p}_{1}^{j}$. We are thus led to consider

$$
\begin{aligned}
F_{g, n}= & \left\langle\left|\prod_{k=1}^{3 g-3+n}\left(\mu_{k} b\right) e^{i\left(\phi_{1}+\phi_{2}\right)}\left(z_{1}\right) e^{-i\left(\phi_{1}+\phi_{2}\right)}\left(z_{2}\right) \prod_{i=1}^{g-1} e^{-\frac{\varphi}{2}} S_{1} e^{\frac{i \sqrt{3}}{2} H}\left(x_{i}\right) \prod_{j=1}^{g-1} e^{-\frac{\varphi}{2}} S_{2} e^{\frac{i \sqrt{3}}{2} H}\left(y_{i}\right)\right|^{2} .\right. \\
& \left.\cdot \prod_{k=1}^{n}\left|e^{-\frac{\varphi}{2}} S^{\mathrm{i}}\right|^{2} \Sigma_{I_{k}}\left(u_{k}\right) \prod_{l=1}^{n}\left|c e^{-\frac{\varphi}{2}} S^{2}\right|^{2} \Sigma_{J_{l}}\left(v_{l}\right)\left|\prod_{a=1}^{3 g-3+n} e^{\varphi} T_{F}\left(r_{a}\right)\right|^{2}\right\rangle
\end{aligned}
$$

where the absolute square takes into account the contribution of the right-moving side and there are implicit world-sheet integrals over $z_{1,2}, x, y, u$. Comparing this expression to [17] we can see that the left moving piece is identical to the supersymmetric contribution of a similar class of $\mathcal{N}=1$ heterotic topological amplitudes. Since left and right movers in (3.6) are essentially identical, we can immediately state the complete result

$$
F_{g, n}=\int_{\mathcal{M}_{(g, n)}}\left\langle\prod_{k=1}^{3 g-3+n}\left|\left(\mu_{k} \cdot G^{-}\right)\right|^{2} \prod_{k=1}^{n} \int \Psi_{I_{k}} \prod_{l=1}^{n} \hat{\Psi}_{J_{l}}\right\rangle_{\mathrm{top}} .
$$

Here again the absolute square takes care of the corresponding right-moving operators and $\hat{\Psi}_{J}=\oint d z \rho(z) \oint d \bar{z} \tilde{\rho}(\bar{z}) \Psi_{J}$ with $\rho$ denoting the unique left moving operator with charge +3 and dimension 0 in the twisted internal theory and $\tilde{\rho}$ denoting the right-moving operator with charge $(-3)$ for IIA and $(+3)$ for IIB. While the operators $\Psi_{I}$ have charge $(-1,+1)$ (and $(-1,-1))$ in IIA (IIB) and dimension $(1,1)$ with respect to the left and right moving $U(1)$ and the twisted Virasoro generators, the operators $\hat{\Psi}_{J}$ have charge $(+2,-2)$ (and $(+2,+2))$ and dimensions $(0,0)$ in IIA (IIB). Note that $S^{\mathrm{i}} \tilde{S}^{\mathrm{i}} \Sigma_{I}$ of the physical vertex is replaced by $\Psi_{I}$ in the twisted theory while $S^{2} \tilde{S}^{\dot{2}} \Sigma_{J}$ is replaced by $\hat{\Psi}_{J}$

The short distance singularity mentioned earlier can arise in general when the operator $\Psi_{I}$ approaches $\hat{\Psi}_{J}$ if the kinetic term $F_{I} \cdot F_{J}$ is non-vanishing. This singularity is of the form $1 /|z|^{2}$ and is due to the exchange of a modulus that couples to $F_{I} \cdot F_{J}$ (see Figure 1). These reducible graphs have to be subtracted in order to compute the effective action terms $F_{g, n}$. However for some special cases one can choose the matter gauge fields in such a way that there are no such reducible graphs:

\footnotetext{
${ }^{3}$ Had we started with the helicity 0 physical vertex operator $S^{(\dot{1}} \tilde{S}^{\dot{2})} \Sigma_{K}$ then this would have given rise to the operator $(\oint d z \rho(z)+\oint d \bar{z} \tilde{\rho}(\bar{z})) \Psi_{K}$ in the twisted theory.
} 
1. When the Calabi-Yau space is an orbifold $\left(T^{2} \times T^{2} \times T^{2}\right) / G$ for some orbifold group $G$ (e.g. $G=\mathbb{Z}_{3}$ ). Denote by $F_{i}$ for $i=1,2,3$ the gauge fields corresponding to the moduli of the three $T^{2}$. The structure constants $C_{i j k}$ are non-zero only if $i, j, k$ are different from each other. This means that the gauge kinetic term $F_{i} . F_{j}$ is non-zero only if $i \neq j$. Choosing all the matter gauge fields coming from the same $T^{2}$ will then have no short distance singularities.

2. A more interesting case is when the type II theory is dual to a heterotic $(4,0)$ compactification. The vector modulus $S$ dual to the heterotic dilaton appears linearly in the prepotential up to exponentially suppressed terms $e^{i S}$. Therefore for large $\operatorname{Im} S$ which corresponds to the weak coupling limit in the heterotic theory, the singularities will disappear.

We should however emphasize that the type II result for $F_{g, n}$ is expected to be exact. This can for example be seen from the effective supergravity action (2.8): The type II dilaton is the lowest component of an $\mathcal{N}=2$ hypermultiplet. Since in standard $\mathcal{N}=2$ superspace the latter cannot appear in chiral F-terms in a consistent manner, it follows that $F_{g, n}$ is independent of the type II dilaton. Therefore it will only receive contributions at the $g$-loop level. Notice that this argument will not go through in the dual heterotic theory since $F_{g, n}$ will indeed have a non-trivial $S$-dependence. Therefore the dual heterotic amplitudes will receive also non-perturbative contributions. However, in order to extract these from the exact type II result one would have to subtract the reducible terms that go like powers of $e^{i S}$.

Notice that (3.7) is a correlator of the twisted internal $\mathcal{N}=2$ SCFT. Depending on whether we consider the A-model or B-model, we have in particular

$$
\begin{array}{ll}
\left|\left(\mu_{k} \cdot G^{-}\right)\right|^{2}=\left(\mu_{k} \cdot G^{-}\right)\left(\bar{\mu}_{k} \cdot \tilde{G}^{+}\right) & \text {A-model } \\
\left|\left(\mu_{k} \cdot G^{-}\right)\right|^{2}=\left(\mu_{k} \cdot G^{-}\right)\left(\bar{\mu}_{k} \cdot \tilde{G}^{-}\right) & \text {B-model }
\end{array}
$$

Near the conifold singularity it is believed that the topological string theory is related to compact $c=1$ matter at self-dual radius coupled to two-dimensional gravity. Indeed it is known that the free energy of the latter reproduces the leading terms of the topological partition function $F_{g}$ near the conifold singularity with the parameter which describes the deformation of the conifold being related to the Legendre transform of the cosmological constant of the two-dimensional gravity. It is then natural to assume that our generalized topological quantities $F_{g, n}$ are related to some deformation of the $c=1$ system coupled to two-dimensional gravity. In the heterotic theory, $F_{g, n}$ have been computed in [1] when all the matter gauge fields are taken from the dilaton multiplet (in the dual type II theory this corresponds to the case (2) discussed above) and it has been shown that near the singularity corresponding to $S U(2)$ enhancement the leading terms are given by the radius deformation of the $c=1$ system coupled to two-dimensional gravity away from the self-dual radius. For completeness we will give a brief review of [1] in the following section. 


\section{The Amplitude in Heterotic String Theory}

We now wish to dualize the results of the previous section to heterotic string theory compactified on $K 3 \times T^{2}$. Applying the same duality mapping as in [9], $F_{g, n}$ for arbitrary $g$ and $n$ are expected to start receiving contributions at the one-loop level. Therefore, in the heterotic weak-coupling regime which we have already considered in the previous section, the amplitude

$$
F_{g, n}=\left\langle V_{(R)}\left(p_{1}\right) V_{(R)}\left(\bar{p}_{2}\right) \prod_{i=1}^{g-1} V_{(T)}\left(\bar{p}_{1}^{i}\right) V_{(T)}\left(\bar{p}_{2}^{i}\right) \prod_{j=1}^{n} V_{(F)}\left(p_{1}^{j}\right) V_{(F)}\left(\bar{p}_{2}^{j}\right)\right\rangle_{1-\mathrm{loop}}^{\text {het }},
$$

is expected to be identical to (3.7). The vertex operators for gravitons, graviphotons and vector partners of the dilaton can for example be found in [9]. Using the same helicity structure and polarizations as in Section 3 we have explicitly

$$
\begin{array}{ll}
\text { gravitons: } & V_{(R)}\left(p_{1}\right)=:\left(\partial Z^{2}-i p_{1} \chi^{1} \chi^{2}\right) \bar{\partial} Z^{2} e^{i p_{1} Z^{1}}:, \\
& V_{(R)}\left(\bar{p}_{1}\right)=:\left(\partial \bar{Z}^{2}-i \bar{p}_{1} \bar{\chi}^{1} \bar{\chi}^{2}\right) \bar{\partial} \bar{Z}^{1} e^{i \bar{p}_{1} \bar{Z}^{1}}:, \\
\text { graviphotons: } & V_{(T)}\left(p_{1}\right)=:\left(\partial X-i p_{1} \chi^{1} \Psi\right) \bar{\partial} Z^{2} e^{i p_{1} Z^{1}}: \\
& V_{(T)}\left(\bar{p}_{1}\right)=:\left(\partial X-i \bar{p}_{1} \bar{\chi}^{1} \Psi\right) \bar{\partial} \bar{Z}^{2} e^{i \bar{p}_{1} \bar{Z}^{1}}: \\
& \\
& V_{(F)}\left(\bar{p}_{1}\right)=:\left(\partial X-i \bar{p}_{1} \bar{\chi}^{1} \Psi\right) \bar{\partial} Z^{2} e^{i \bar{p}_{1} \bar{Z}^{1}}: \\
& V_{(F)}\left(p_{1}\right)=:\left(\partial X-i p_{1} \chi^{1} \Psi\right) \bar{\partial} \bar{Z}^{2} e^{i p_{1} Z^{1}}:
\end{array}
$$

These vertices are written in the (0)-ghost picture using the same complex basis for the space-time coordinates $\left(Z^{1}, \bar{Z}^{1}, Z^{2}, \bar{Z}^{2}\right)$ and their fermionic partners $\left(\chi^{1}, \bar{\chi}^{1}, \chi^{2}, \bar{\chi}^{2}\right)$ as in Section 3. The (complex) coordinate on $T^{2}$ is called $(X, \bar{X})$ with its superpartner $(\Psi, \bar{\Psi})$.

In order to compute (4.1) it suffices to consider the odd spin-structure since the even one gives essentially the same result. In this case both gravitons need to contribute the fermion bilinear piece in order to soak up the corresponding zero modes of the space-time fermions. Due to the presence of a Killing spinor on the world-sheet torus (see [9]), we pick one of the graviphotons to be inserted in the $(-1)$ picture

$$
V_{(T)}^{(-1)}\left(p_{1}\right)=e^{-\phi} \Psi \bar{\partial} Z^{2} e^{i p_{1} Z^{1}},
$$

which will be accompanied by a picture changing operator. The latter, in order to soak the $\Psi$ zero-mode needs to contribute $e^{\phi} \bar{\Psi} \partial X$. Since the remaining $\Psi$ cannot be contracted, it follows that all gauge-field vectors will contribute their $\partial X$ piece, which will just give zero modes. Therefore, the only remaining non-trivial piece is the correlator of the space-time bosons. Extracting the appropriate piece proportional to $p_{1}^{2} \bar{p}_{1}^{2} \prod_{i=1}^{g-1} p_{1}^{i} \bar{p}_{1}^{i} \prod_{j=1}^{n} \bar{p}_{1}^{j} p_{1}^{j}$ it takes the form

$$
\mathcal{C}_{g, n}=\left\langle\prod_{i=1}^{g} \int d^{2} x_{i} Z^{1} \bar{\partial} Z^{2}\left(x_{i}\right) \int d^{2} y_{i} \bar{Z}^{1} \bar{\partial} \bar{Z}^{2}\left(y_{i}\right) \prod_{j=1}^{n} \int d^{2} u_{j} \bar{Z}^{1} \bar{\partial} Z^{2}\left(u_{j}\right) \int d^{2} v_{j} Z^{1} \bar{\partial} \bar{Z}^{2}\left(v_{j}\right)\right\rangle .
$$


Since this is just a free field correlator, it can be computed by evaluating the generating functional

$$
\begin{aligned}
& G\left(\epsilon_{-}, \epsilon_{+}\right)=\sum_{g=1}^{\infty} \sum_{n=1}^{\infty} \frac{\epsilon_{-}^{2 g} \epsilon_{+}^{2 n} \mathcal{C}_{g, n}}{(g ! n !)^{2} \tau_{2}^{2 g+2 n}}= \\
& =\frac{\int \prod_{i=1}^{2} \mathcal{D} Z^{i} \mathcal{D} \bar{Z}^{i} \operatorname{Exp}\left(-S+\frac{\epsilon_{-}}{\tau_{2}} \int\left(Z^{1} \bar{\partial} Z^{2}+\bar{Z}^{2} \bar{\partial} \bar{Z}^{1}\right)+\frac{\epsilon_{+}}{\tau_{2}} \int\left(\bar{Z}^{1} \bar{\partial} Z^{2}+\bar{Z}^{2} \bar{\partial} Z^{1}\right)\right)}{\int \prod_{i=1}^{2} \mathcal{D} Z^{i} \mathcal{D} \bar{Z}^{i} \operatorname{Exp}(-S)}
\end{aligned}
$$

where $S$ is the usual free-field action $S=\sum_{i=1,2} \frac{1}{\pi} \int d^{2} x\left(\partial Z^{i} \bar{\partial} \bar{Z}^{i}+\partial \bar{Z}^{i} \bar{\partial} Z^{i}\right)$ and $\epsilon_{ \pm}$are two arbitrary parameters. Notice that all terms in (4.3) are bilinear in the fields. Thereby the functional integral is Gaussian and can be evaluated explicitly. This is essentially done in the same way as in [1, 9] and we can therefore immediately state the result

$$
G\left(\epsilon_{-}, \epsilon_{+}\right)=\frac{2 \pi i\left(\epsilon_{-}+\epsilon_{+}\right) \bar{\eta}^{3}}{\bar{\vartheta}_{1}\left(\epsilon_{-}+\epsilon_{+}, \bar{\tau}\right)} \cdot \frac{2 \pi i\left(\epsilon_{-}-\epsilon_{+}\right) \bar{\eta}^{3}}{\bar{\vartheta}_{1}\left(\epsilon_{-}-\epsilon_{+}, \bar{\tau}\right)} e^{-\frac{\pi}{\tau_{2}}\left(\epsilon_{+}^{2}+\epsilon_{-}^{2}\right)}
$$

Upon introducing also a generating functional for the amplitudes

$$
F\left(\epsilon_{-}, \epsilon_{+}\right)=\sum_{g=1}^{\infty} \sum_{n=1}^{\infty} \epsilon_{-}^{2 g} \epsilon_{+}^{2 n} F_{g, n}
$$

we can state the final result

$F\left(\epsilon_{-}, \epsilon_{+}\right)=\int \frac{d^{2} \tau}{\tau_{2}} \frac{\bar{E}_{4} \bar{E}_{6}}{\bar{\eta}^{24}} \sum_{\Gamma^{(2,2)}}\left(\frac{2 \pi i\left(\epsilon_{-}+\epsilon_{+}\right) \bar{\eta}^{3}}{\bar{\vartheta}_{1}\left(\tilde{\epsilon}_{-}+\tilde{\epsilon}_{+}, \bar{\tau}\right)}\right)\left(\frac{2 \pi i\left(\epsilon_{-}-\epsilon_{+}\right) \bar{\eta}^{3}}{\bar{\vartheta}_{1}\left(\tilde{\epsilon}_{-}-\tilde{\epsilon}_{+}, \bar{\tau}\right)}\right) e^{-\frac{\pi}{\tau_{2}}\left(\tilde{\epsilon}_{-}^{2}+\tilde{\epsilon}_{+}^{2}\right)} q^{\frac{1}{2}\left|P^{L}\right|^{2}} \bar{q}^{\frac{1}{2}\left|P^{R}\right|^{2}}$

where we have introduced $\tilde{\epsilon}_{ \pm}=\epsilon_{ \pm} \tau_{2} P^{L} / \sqrt{(T-\bar{T})(U-\bar{U})}$ with $P^{L}$ and $P^{R}$ the lattice momenta of the internal $\Gamma^{(2,2)}$ lattice, while $q=e^{2 \pi i \tau}$. Notice that we have written this amplitude at a point in moduli space with no Wilson lines in the right moving sector being switched on. $P_{L}$ is the central charge of the $\mathcal{N}=2$ algebra and is given by

$$
P^{L}=\frac{1}{\sqrt{(T-\bar{T})(U-\bar{U})}}\left(n_{1}+n_{2} \bar{T} \bar{U}+m_{1} \bar{T}+m_{2} \bar{U}\right)
$$

where $m_{1}, m_{2}, n_{1}, n_{2}$ are integers and $T$ and $U$ are the Kähler and complex structure moduli of the compactification torus.

Near an $S U(2)$ enhanced symmetric point in moduli space $P_{L} \rightarrow 0$ for two lattice points in $\Gamma^{(2,2)}\left(n_{1}=n_{2}=0, m_{1}=-m_{2}= \pm 1\right)$. In the vicinity of these points the leading singularity for $F_{g, n}$ can be easily computed from (4.6) as $\epsilon_{-}^{2 g} \epsilon_{+}^{2 n}$ term in the expansion of

$$
F \sim \int \frac{d \tau_{2}}{\tau_{2}} \frac{\pi \epsilon_{1}}{\sin \left(\pi \epsilon_{1} \tau_{2}\right)} \frac{\pi \epsilon_{2}}{\sin \left(\pi \epsilon_{2} \tau_{2}\right)} e^{-\tau_{2} \mu}
$$


where $\mu=\sqrt{\frac{i}{\pi}} \sqrt{(T-\bar{T})(U-\bar{U})} \bar{P}^{L} \sim \sqrt{\frac{i}{\pi}}(T-U)$ and $\epsilon_{1}=\epsilon_{+}+\epsilon_{-}$and $\epsilon_{2}=\epsilon_{+}-\epsilon_{-}$ and we have also rescaled $\tau_{2} \bar{\mu} \rightarrow \tau_{2}$. The leading singularity as $\mu \rightarrow 0$ in the $\epsilon_{-}^{2 g} \epsilon_{+}^{2 n}$ term is given by $\mu^{2-2 g-2 n}$. Note that this is holomorphic in the relevant vector modulus $T-U$ and it is related to the fact that our vertices for the graviphoton and dilaton field strengths involve only $\partial X$ in the $T^{2}$ direction which contributes $P_{L}$ in the correlation function. This expression is just the free energy of the $c=1$ system coupled to 2-dimensional gravity with the cosmological constant $\mu$ and at radius $\left|\epsilon_{1} / \epsilon_{2}\right|$ in units where the self dual radius is 1 [7]. Note that for $\epsilon_{+}=0$, which reduces to the standard $F_{g}$, we have $\epsilon_{2}=-\epsilon_{1}$ giving rise to the free energy of the $c=1$ system at the self-dual radius. Equation (4.8) exhibits certain symmetries generated by $\left(\epsilon_{1}, \epsilon_{2}\right) \rightarrow\left(-\epsilon_{1}, \epsilon_{2}\right)$ and $\left(\epsilon_{1}, \epsilon_{2}\right) \rightarrow\left(\epsilon_{2}, \epsilon_{1}\right)$. 4 In particular this implies that (4.8) is invariant under the exchange of $\epsilon_{+}$and $\epsilon_{-}$and is even in $\epsilon_{1}, \epsilon_{2}$ as well as $\epsilon_{+}$and $\epsilon_{-}$. The latter is to be expected since $\epsilon_{+}$and $\epsilon_{-}$couple to self-dual and anti-self dual field strengths and a Lorentz invariant string effective action must contain even numbers of self-dual and anti-selfdual tensors.

This expression is very similar to the perturbative part of the free energy of the pure $\mathcal{N}=2 S U(2)$ Yang-Mills theory in the $\Omega$-background given in (A.7) of [18] with the identification $2 \pi i \epsilon_{1,2} \rightarrow \epsilon_{1,2}$. There instead of the sin factors in the denominator of (4.8) they have the factor

$$
F \sim \int \frac{d \tau_{2}}{\tau_{2}} \frac{\pi \epsilon_{1}}{\sin \left(\pi \epsilon_{1} \tau_{2}\right)} \frac{\pi \epsilon_{2}}{\sin \left(\pi \epsilon_{2} \tau_{2}\right)} e^{-\tau_{2} \mu} e^{-i \pi\left(\epsilon_{1}+\epsilon_{2}\right) \tau_{2}}
$$

This expression has only the symmetry $\epsilon_{1} \leftrightarrow \epsilon_{2}$. It is even in $\epsilon_{-}$but not in $\epsilon_{+}$due to the extra phase $e^{-i \pi\left(\epsilon_{1}+\epsilon_{2}\right) \tau_{2}}$.

\section{Concluding remarks}

In this paper we have analyzed the $\mathcal{N}=2$ F-terms of the type $W^{2 g} \Upsilon^{n}$ where $W$ is the chiral Weyl superfield and $\Upsilon$ are chiral projectors of real functions of vector multiplets. These terms give rise to couplings of the form $\left(R^{-}\right)^{2}\left(T^{-}\right)^{2 g-2}\left(F^{+}\right)^{2 n}$ with $R, T$ and $F$ being the Riemann tensor, graviphoton and matter vector field strengths. In the special case where the vector multiplets only involve the heterotic dilaton $S$, these amplitudes have been computed at one loop in heterotic string [1] where it was shown that in the field theory limit they are given by the radius deformation of $c=1$ matter coupled to 2-dimensional gravity. This result is perturbative in the heterotic dilaton and should receive non-perturbative corrections. In the present work we have constructed these amplitudes in type II theory for general $\Upsilon$ involving arbitrary vector multiplets, where they appear at genus $g$ and are given by certain correlation functions in the twisted topological theory which is essentially the square of the supersymmetric sector of the $\mathcal{N}=1$ heterotic topological amplitude constructed earlier in [17]. Due to the fact that the type II dilaton is a hypermultiplet we expect this genus $g$ result to be exact.

\footnotetext{
${ }^{4}$ The resulting symmetry group is a discreet non-abelian group Dihedral ${ }_{4}$ of 8 elements $\left( \pm 1, \pm \sigma_{1}, \pm \sigma_{3}, \pm i \sigma_{2}\right)$.
} 
Remarkably, the field theory limit in the heterotic side (when the matter vector fields are taken from dilaton multiplet) reproduces the perturbative part of Nekrasov results for the $\mathcal{N}=2$ gauge theories in the general $\Omega$ background involving 2 complex parameters $\epsilon_{ \pm}$, up to a phase depending on $\epsilon_{+}$, which might be attributable to different regularization schemes. In string theory $\epsilon_{-}$couples to the graviphoton $T^{-}$and $\epsilon_{+}$to $F_{S}^{+}$. It might appear strange that while $\epsilon_{-}$couples to the anti-self dual graviphoton, $\epsilon_{+}$couples to the self-dual dilaton vector. However in the heterotic theory this is quite natural for two reasons:

1. The left moving (i.e. in the supersymmetric sector) the vertices of the anti-self dual graviphoton and the self-dual dilaton vector are identical and in fact both of them contribute $P_{L}$ lattice momentum. The difference in the two vertices appears in the bosonic sector where they give anti-self dual and self dual Lorentz currents respectively. Thus setting $\epsilon_{+}= \pm \epsilon_{-}$(i.e. $\epsilon_{1}$ or $\epsilon_{2}$ equal to zero) we find a manifest $S O(2)$ invariance as happens in the gauge theory.

2. More importantly, since the anti-selfdual graviphoton and selfdual dilaton vector vertices contribute $P_{L}$, this amplitude carries $(2 g+2 n-2) P_{L}$. Therefore, as discussed in the last section, near the $S U(2)$ enhancement $\mu \rightarrow 0$, where $\mu$ is the holomorphic vector modulus, the leading singularity has the behaviour $\mu^{2-2 g-2 n}$ exactly as in the gauge theory. For example, if some of the vertices had been that of selfdual graviphoton, they would have contributed $\bar{P}_{L}$, and this would have given rise to factors of $1 / \bar{\mu}$ to the singularity. The answer then would not be holomorphic in vector moduli as opposed to the gauge theory result of [10, 18] as expected from the fact that this result gives the prepotential of the gauge theory.

This generalizes the case $\epsilon_{+}=0$ for which it is known that $F_{g, 0}$, in the field theory limit and weak heterotic coupling, reproduces the perturbative part of Nekrasov's result. For $\epsilon_{+} \neq 0$, while the heterotic string (as well as the type II) result is even in both $\epsilon_{+}$and $\epsilon_{-}$ (this is a consequence of the Lorentz invariance of the string effective action), Nekrasov's result is even only in $\epsilon_{-}$and due to the extra phase in (4.9) it contains all powers in $\epsilon_{+}$. On the other hand, the gauge theory action given in eqs. (2.1) and (2.3) of [18] is Lorentz invariant provided that transformations of the $\mathcal{N}=2$ gauge theory fields are compensated by a simultaneous Lorentz transformation of the $\Omega$ background. One would therefore expect that the quantum corrections to the prepotential must also have this symmetry. Since $\epsilon_{ \pm}$transform under the $S U(2)_{L} \times S U(2)_{R}$ of the Lorentz group, the invariants must be quadratic in $\epsilon_{+}$as well as $\epsilon_{-}$. The appearance of odd powers in $\epsilon_{+}$in the gauge theory result is therefore surprising and might be a consequence of the regularization procedure. In fact the generalized Gopakumar-Vafa formula (eq.(2.6) of [16] ) is based on the one loop Schwinger result of a particle in representation $\mathcal{R}$ of $S U(2)_{L} \times S U(2)_{R}$ in the background of constant gauge field strength $F=F_{1} d x^{1} \wedge d x^{2}+F_{2} d x^{3} \wedge d x^{4}$ :

$$
\int \frac{d s}{s} \frac{\operatorname{Tr}_{\mathcal{R}}(-1)^{\sigma_{L}+\sigma_{R}} e^{-s m^{2}} e^{-2 s e\left(\sigma_{L} F_{+}+\sigma_{R} F_{-}\right)}}{\left(2 \sinh \left(s e F_{1} / 2\right)\right)\left(-2 \sinh \left(s e F_{2} / 2\right)\right.},
$$

where $F_{ \pm}$are the selfdual and anti-selfdual parts of $F, \sigma_{L, R}$ are the weights of the $S U(2)_{L, R}$ in the representation $\mathcal{R}$ and $m$ and $e$ are the particle's mass and charge respectively. In 
each representation $\mathcal{R}=\left(j_{L}, j_{R}\right)$ the above expression is even in $F_{+}$and $F_{-}$since in the trace, $\sigma_{L, R}=-j_{L, R}, \ldots, j_{L, R}$. This is consistent with the result of the heterotic string as $F_{ \pm}$in (5.1) are related to the background $\epsilon_{ \pm}$.

The type II result we have obtained in this paper, when applied for the vector multiplets corresponding to the dual heterotic dilaton $S$, also captures the non-perturbative contributions that go like exponentials of $S$. As pointed out in Section 3, in order to extract the 1PI effective action term, one needs to subtract the reducible graph shown in fig.1. Note that unlike the heterotic case, the type II result is not symmetric under the exchange of $\epsilon_{+}$and $\epsilon_{-}$. Indeed, while the power of $\epsilon_{-}$counts twice the genus $g$ of the Riemann surface, $\epsilon_{+}$counts the number of vertices $2 n$ in the twisted theory. This is also true in the gauge theory result (at the non-perturbative level). It will be interesting to see how the type II amplitudes considered here are related to the generalized GopakumarVafa formula [16] and to non-perturbative part in the gauge theory prepotential [10, 18] in the presence of arbitrary $\epsilon_{+}$and $\epsilon_{-}$.

In the heterotic side, near the enhanced symmetric point, the leading singularity is given by the free energy of the radius deformed (away from the self-dual radius) $c=1 \mathrm{CFT}$ coupled to 2-dimensional gravity. By heterotic - type II duality, the type II amplitudes we have considered, when specialized to all the matter vector fields to be the heterotic dilaton vector fields, near the conifold should also describe the radius deformation of the above system. While in the type II it is expected that turning on $\epsilon_{+}$should be some deformation of the self-dual $c=1$ system coupled to 2-dimensional gravity, it will be interesting to establish directly in type II theory (i.e. without resorting to heterotic duality) that the deformation is given by the change of radius.

\section{Acknowledgements}

We would like to thank S. Shatashvili for enlightening discussions and N. Nekrasov for helpful correspondence. I.A. acknowledges also discussions with A. Gerasimov. Work supported in part by the European Commission under the ERC Advanced Grant 226371 and the contract PITN-GA-2009-237920 and in part by the CNRS grant GRC APIC PICS 3747. The research of S.H. has been supported by the Swiss National Science Foundation. T.R.T. is grateful to CERN Theory Unit for financial support and kind hospitality during the first part of his sabbatical leave. His research is partially supported by the U.S. NSF Grants PHY-0600304, PHY-0757959. Any opinions, findings, and conclusions or recommendations expressed in this material are those of the authors and do not necessarily reflect the views of the U.S. National Science Foundation.

\section{References}

[1] J. F. Morales and M. Serone, Nucl. Phys. B 481 (1996) 389 arXiv:hep-th/9607193.

[2] N. Seiberg and E. Witten, Nucl. Phys. B 431 (1994) 484. 
[3] S. Kachru and C. Vafa, Nucl. Phys. B 450 (1995) 69 arXiv:hep-th/9505105; S. Ferrara, J.A. Harvey, A. Strominger and C. Vafa, Phys. Lett. B 361 (1995) 59 arXiv:hep-th/9505162.

[4] A. Strominger, Nucl. Phys. B 451 (1995) 96 arXiv:hep-th/9504090.

[5] I. Antoniadis, E. Gava, K. S. Narain and T. R. Taylor, Nucl. Phys. B 413 (1994) 162 arXiv:hep-th/9307158.

[6] M. Bershadsky, S.Cecotti, H. Ooguri and C. Vafa, Commun. Math. Phys. 165 (1994) 311 [arXiv:hep-th/9309140].

[7] I. Klebanov and D. Gross, Nucl. Phys. B 344 (1990) 475; J. Distler and C. Vafa, Mod. Phys. Lett. A 6 (1991) 259; S. Mukhi and C. Vafa, Nucl. Phys. B 407 (1993) 667; (1991) 259; D. Ghoshal, C. Imbimbo and S. Mukhi, Nucl. Phys. B 440 (1995) 355.

[8] D. Ghoshal and C. Vafa, Nucl. Phys. B 453 (1995) 121 arXiv:hep-th/9506122.

[9] I. Antoniadis, E. Gava, K. S. Narain and T. R. Taylor, Nucl. Phys. B 455 (1995) 109 arXiv:hep-th/9507115].

[10] N. A. Nekrasov, Adv. Theor. Math. Phys. 7 (2004) 831 arXiv:hep-th/0206161.

[11] G. W. Moore, N. Nekrasov and S. Shatashvili, Commun. Math. Phys. 209 (2000) 97 arXiv:hep-th/9712241.

[12] A. Lossev, N. Nekrasov and S. L. Shatashvili, In "Cargese 1997, Strings, branes and dualities" 359-372. arXiv:hep-th/9801061

[13] R. Gopakumar and C. Vafa, arXiv:hep-th/9809187.

[14] R. Gopakumar and C. Vafa, arXiv:hep-th/9812127.

[15] T. J. Hollowood, A. Iqbal and C. Vafa, JHEP 0803 (2008) 069 arXiv:hep-th/0310272.

[16] A. Iqbal, C. Kozcaz and C. Vafa, JHEP 0910 (2009) 069 arXiv:hep-th/0701156]. .

[17] I. Antoniadis, E. Gava, K. S. Narain and T. R. Taylor, Nucl. Phys. B 476 (1996) 133 arXiv:hep-th/9604077.

[18] N. Nekrasov and A. Okounkov, arXiv:hep-th/0306238.

[19] M. de Roo, J. W. van Holten, B. de Wit and A. Van Proeyen, Nucl. Phys. B 173 (1980) 175. 